Abstract: amounts of radiation. Understanding their behavior in such an event is important in determining whether they would still function properly. We built a SPICE model of the silicon strip module electrical system to determine the behavior of its elements during a
realistic beam loss scenario. We found that the power supply and bias filter characteristics strongly affect the module response in such scenarios. In particular, the following self-limiting phenomena were observed: there is a finite amount of charge initially available on the bias filter capacitors for collection by the strips; the power supply current limit reduces the rate at which the bias filter capacitors effects provide a larger measure of safety during beam loss events than we have previously assumed.

The ATLAS SCT Barrel Module:

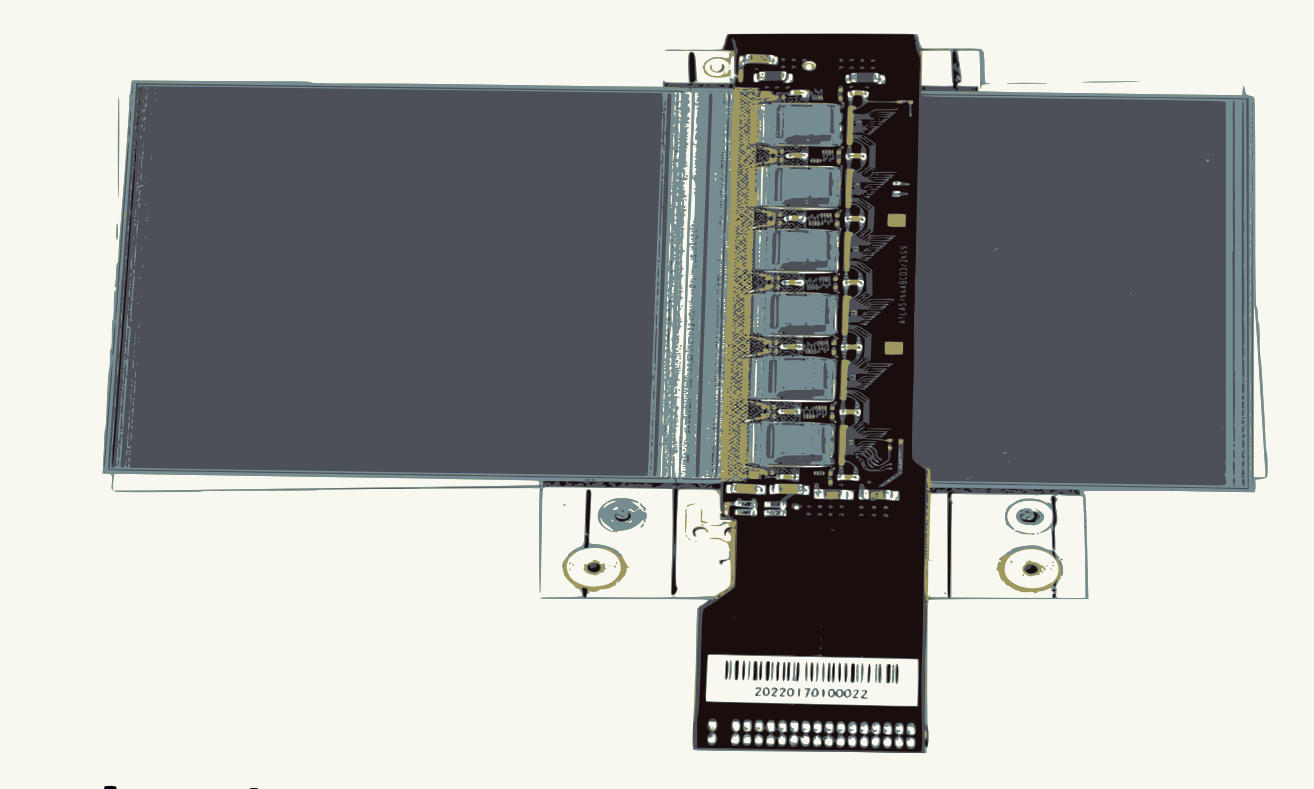

\section{Block Diagram}

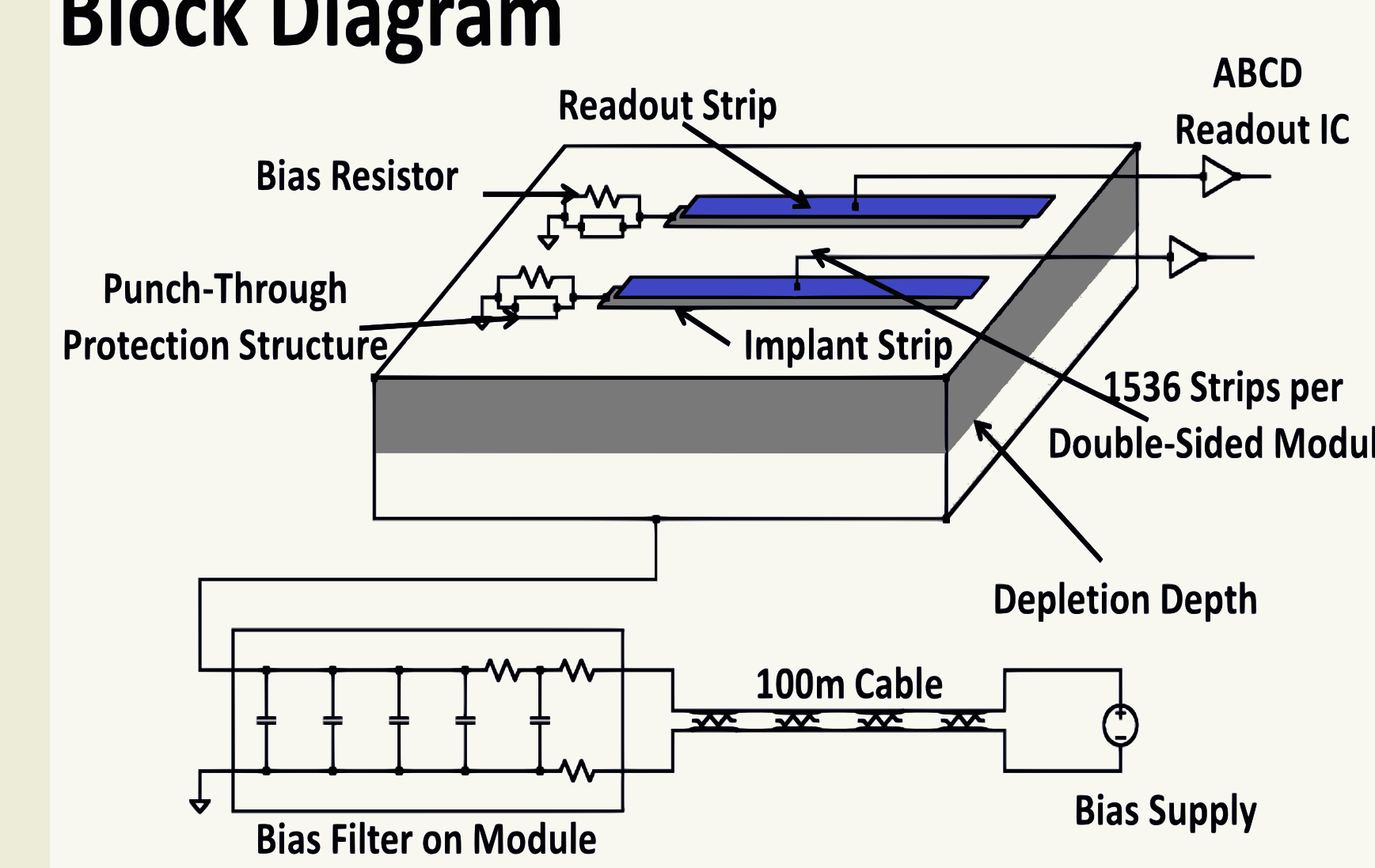

The ATLAS [1] SemiConductor Tracker (SCT) is one component of the ATLAS Inner Detector, which tracks charged particles resulting from endcap regions, with 2112 modules comprising the barrel region. There are four silicon strip sensors per double-sided barrel module, each containing chained together on the readot electo a to make an effective combination of $153612-\mathrm{cm}$ strips per module. Previous simulations have
shown that during the most likely beam loss scenarios, the SCT barrel les may experience a large particle flux of up to $5.4 \times 10^{5} \mathrm{MIPs}$ per strip per bunch crossing [3]. All components inside the detector volume
have been thoroughly tested for radiation hardness such that the extra have been thoroughly tested for radiation hardness such that the extra radiation damage from a beam loss can be tolerated. However, both the
ABCD readout IC [4] and the coupling capacitors between the implant and readout strips may experience problems from a large instantaneous charge
deposition. The ABCD is rated to handle up to $5 \mathrm{nC}$ in $25 \mathrm{~ns}$, but previous coupling capacitor is rated to $100 \mathrm{~V}$, but again, higher values have been observed with no apparent damage $[5,6]$. However, since all sensors have
only been systematically tested up to $100 \mathrm{~V}$, this is still considered the safe limit. Here, we simulate the response of the ATLAS barrel modules to the to be performed, and we determine what conditions are necessary to exceed

SPICE Simulation Circuit Diagrams:
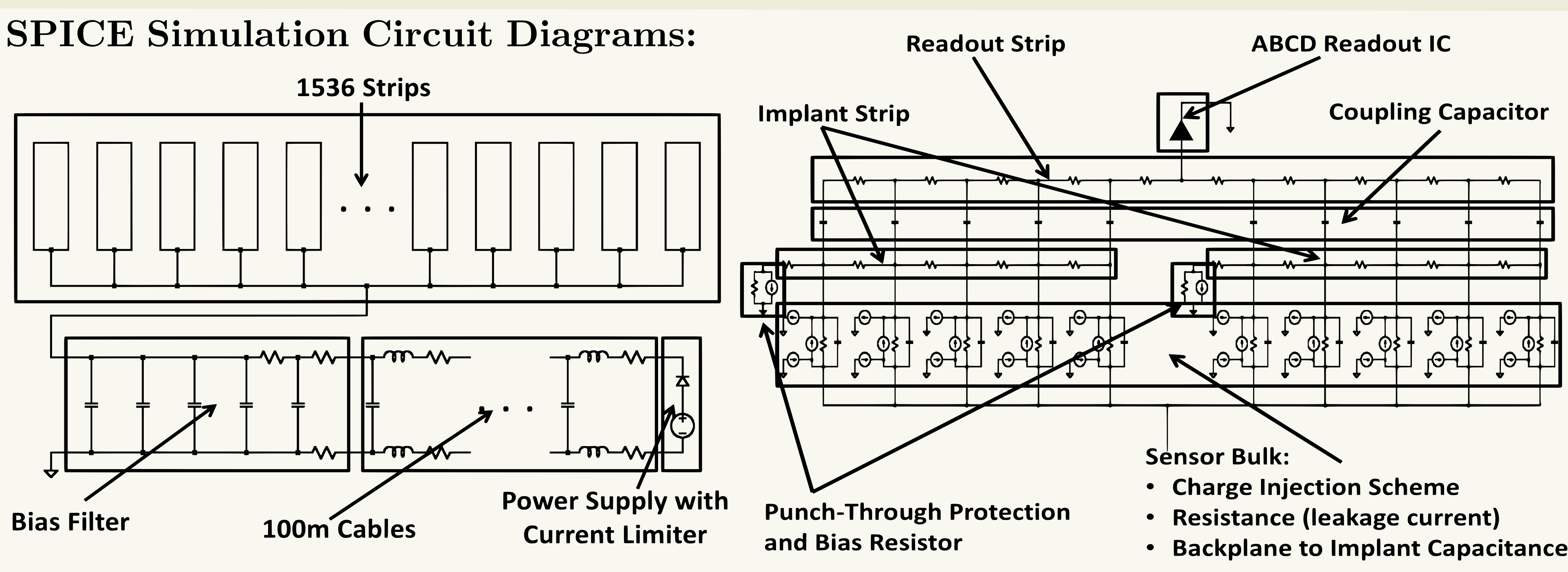

simulated 1536 individual strips, plus the power sup
sur

A Detailed Look at Components of Our Model: ABCD Readout IC:

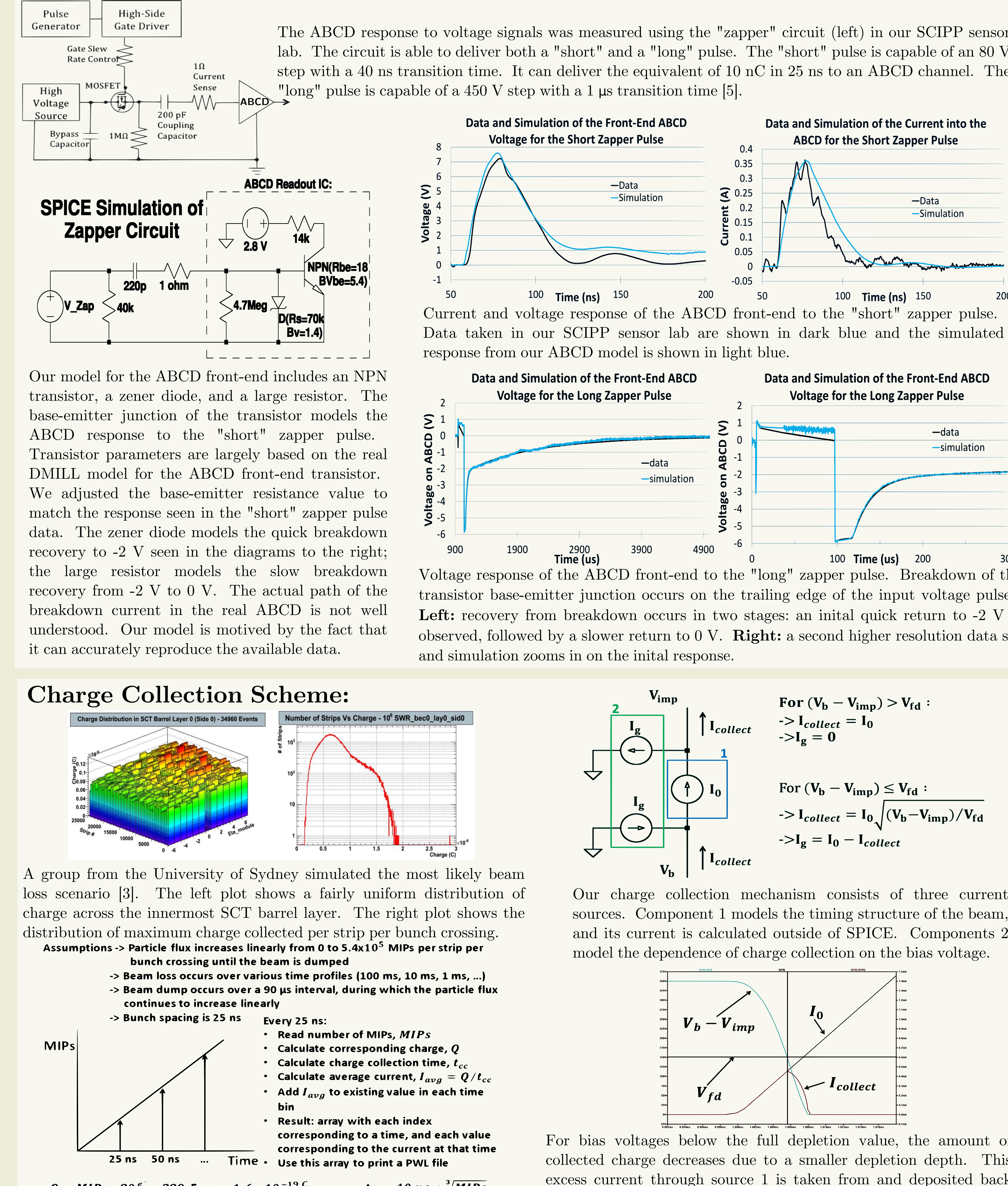

Punch-Through Protection:

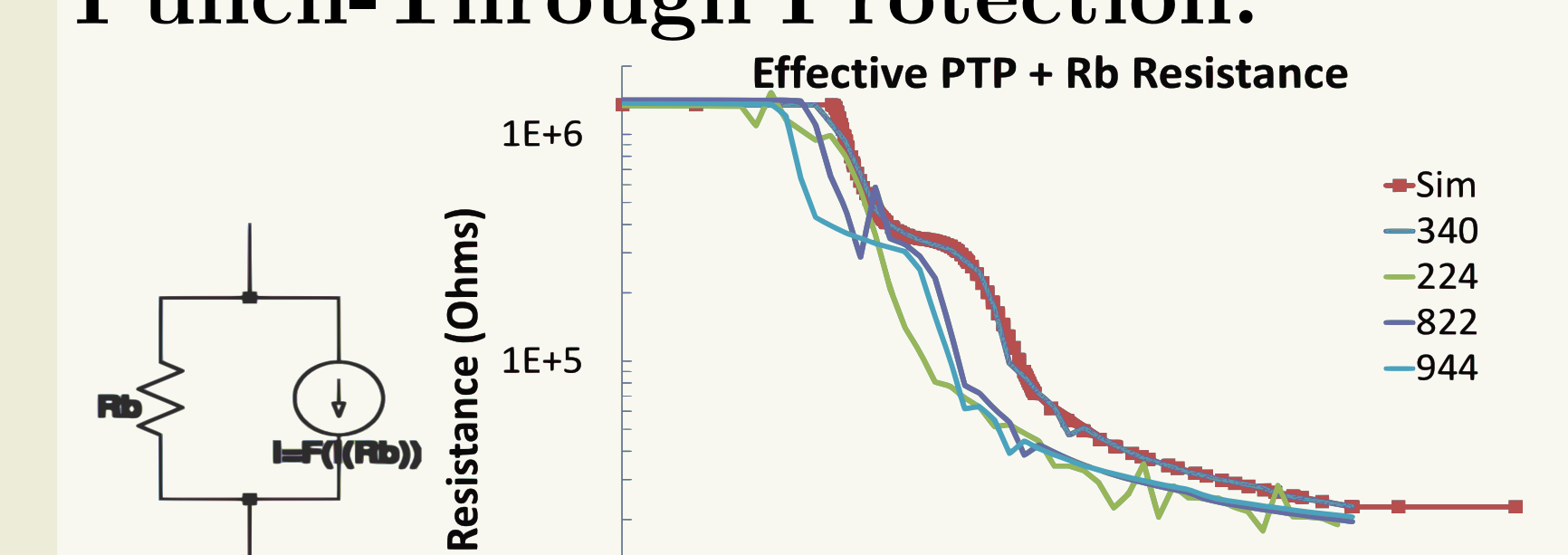

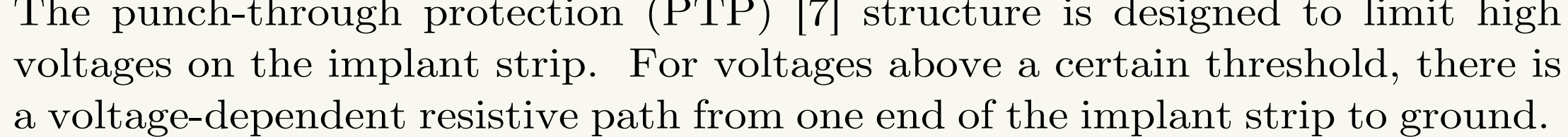

Results:

$10 \mathrm{~ms}$ Beam Loss Scenario for Non-Irradiated Sensors:

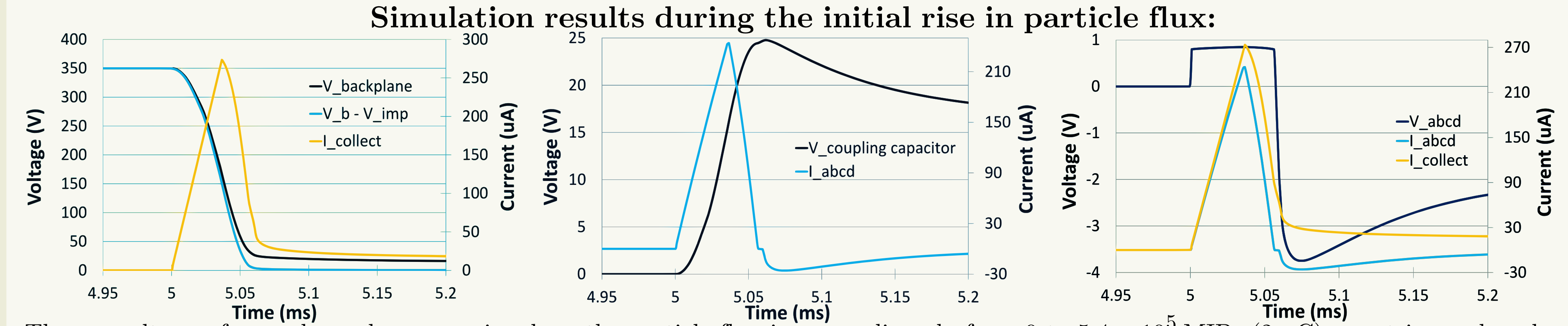

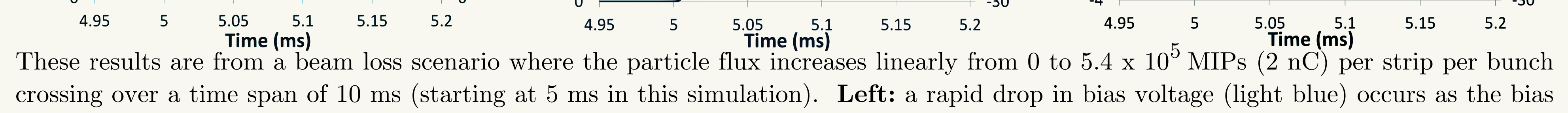

crossing over a time span of $10 \mathrm{~ms}$ (starting at $5 \mathrm{~ms}$ in this simulation). Leftt: a rapid drop in bias voltage (light blue) occurs as the bian
filter capacitors are drained of their charge. This drop in bias voltage leads to a smaller depletion depth in the sensor. Consequently, the

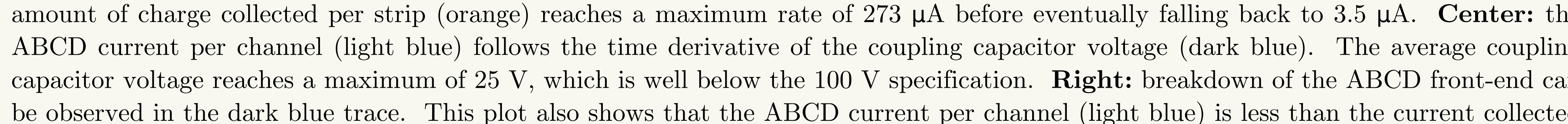

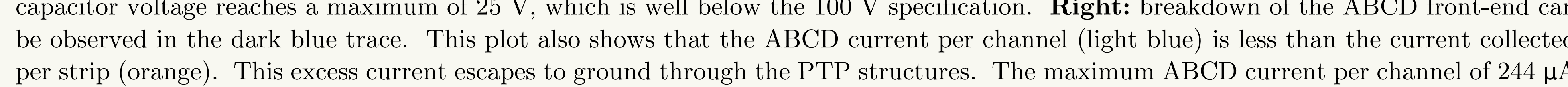
corresponds to $6.1 \mathrm{pC}$ of charge in $25 \mathrm{~ns}$, which is well below the $5 \mathrm{nC}$ in $25 \mathrm{~ns}$ spect

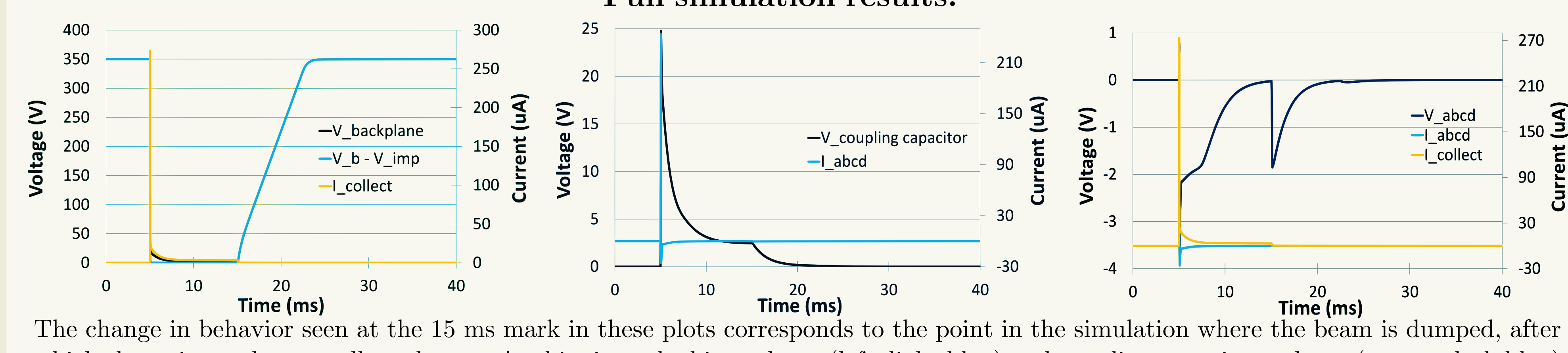

(n) which the strips no longer collect charge. At this time, the bias voltage (left, light blue) and coupling capacitor voltage (center, dark blue Summary of Beam Loss Simulations Over Various Time Profiles:

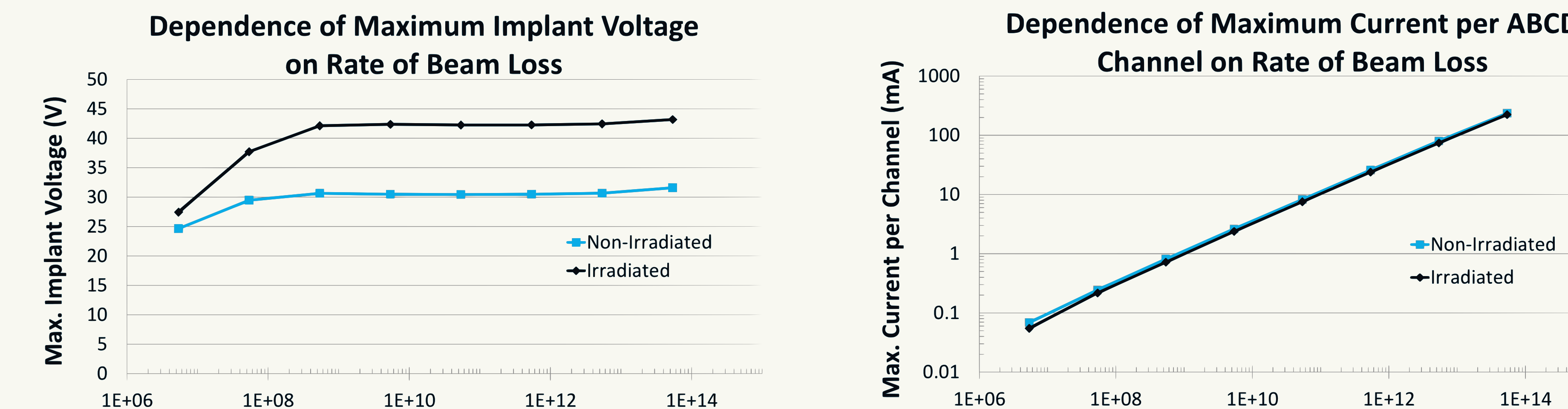

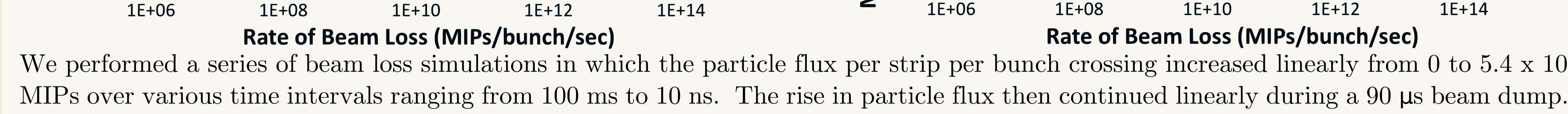

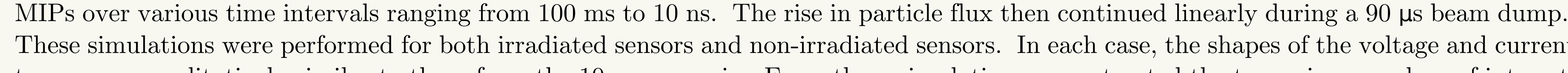
traces were qualitatively similar to those from the $10 \mathrm{~ms}$ scenario. From these simulations, we extracted the two primary values of interesty
the maximum coupling capacitor voltage on the node furthest from the PTP and the maximum current per channel into the ABCD frontend. These are shown in the two summary plots above. Left: the maximum implant voltage reaches a plateau, which varies with the
operational bias voltage, due to the finite amount of charge stored on the bias filter capacitors. Right: the maximum current per ABCD Simulation of Laser Pulse Studies:

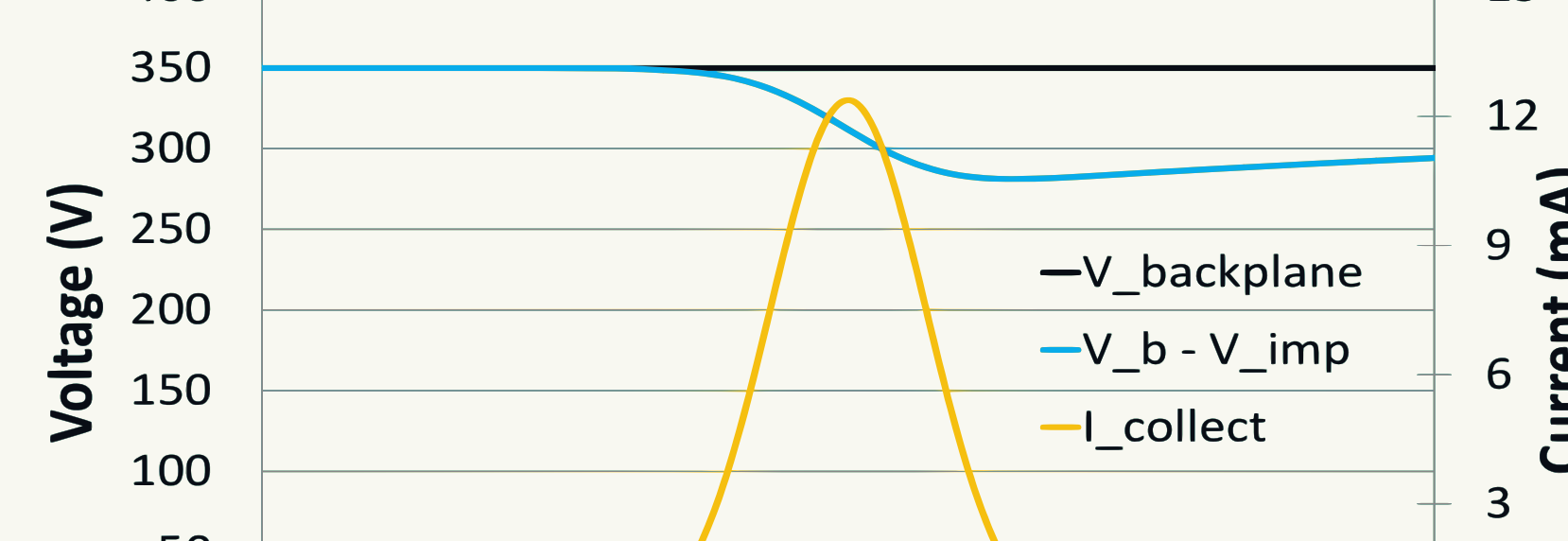

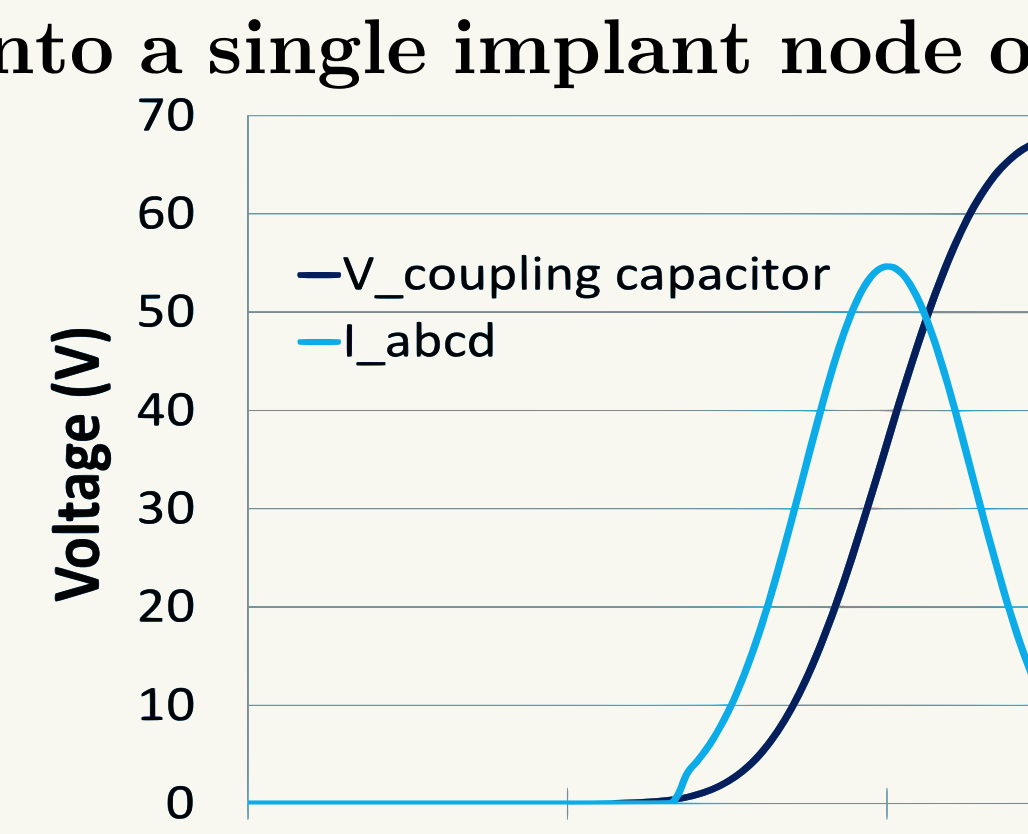
$T_{\text {Timé }}^{2}(\mu s)$
Time $^{2}$ ( $\mu$ s)

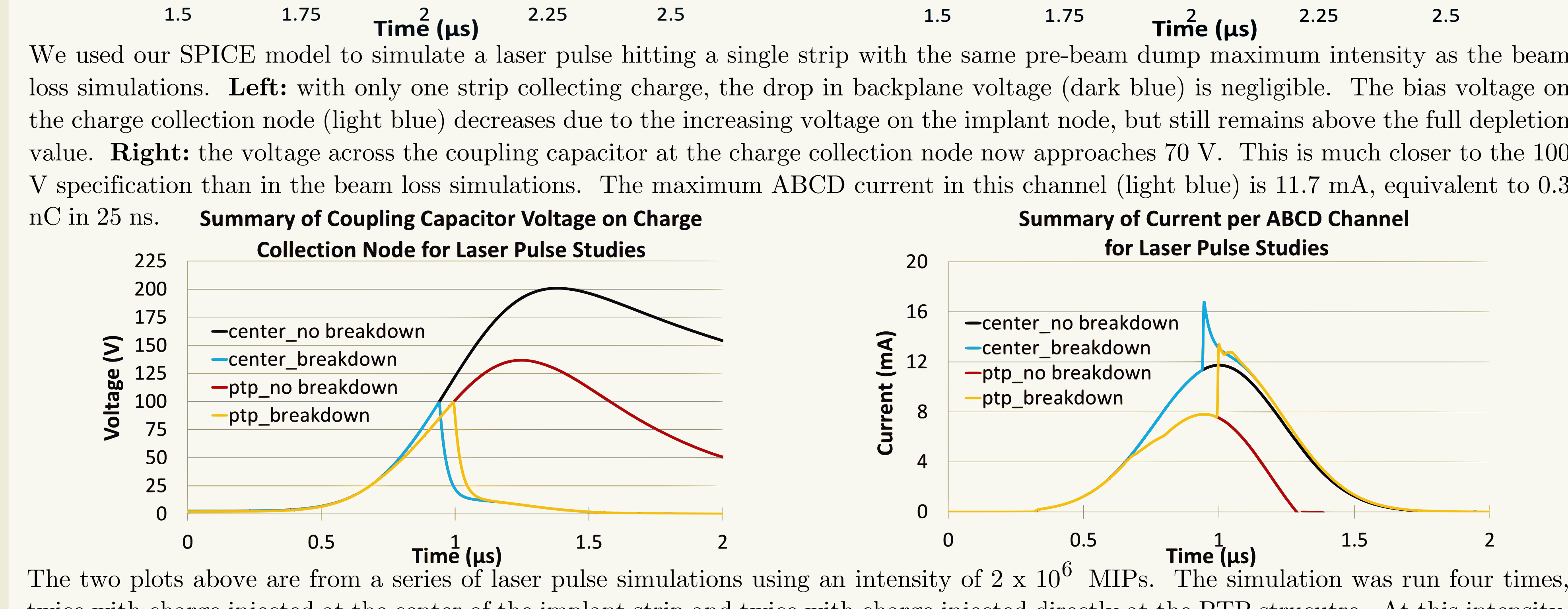

twice with charge injected at the center of the implant strip and twice with charge injected directly at the PTP strucutre. At this intensity,
the mechanism that shorts the imp the $100 \mathrm{~V}$ breaklowis specification, so two of the simulations were run with an additional breakdow.

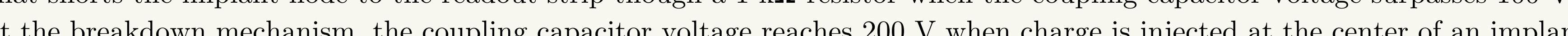
strip (dark blue), and it reaches $137 \mathrm{~V}$ when charge is injected directly at the PTP structure (red). Right: when the coupling capacito Conclusions and Future Work:

\section{Our simulations show that during the most like}

module is rather uniform, there exist inherent self-protection features in the barrel module electrical system. These self-protoction
features are the finite amount of charge stored on the bias filter capacitors and the power supply current limit. Beccuse of these
feates

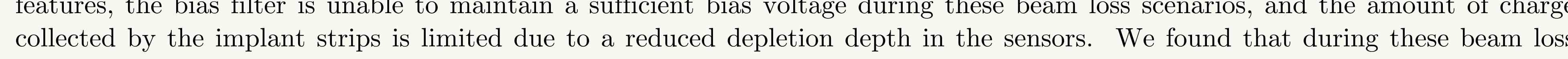

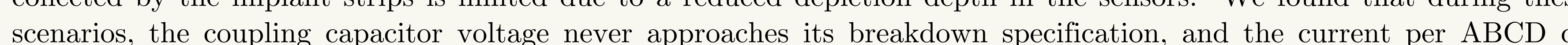
reaches its specified limit for beam loss rates in excess of $5.4 \times 10^{13} \mathrm{MIPs} / \mathrm{bunch} /$ second. In cases where only y fraction of the strips $\mathrm{or}$. features are not actuated, and the observed behavior of the module is very different. Therefore, these sorts of studies may not be an effective way to evaluate potential damage of SCI strips durningenes beste
strips in a single SCT module may exist, it would be useful to investigate what effect, if any, these variations have on the observed References:

2008 JINST 3 S08003.

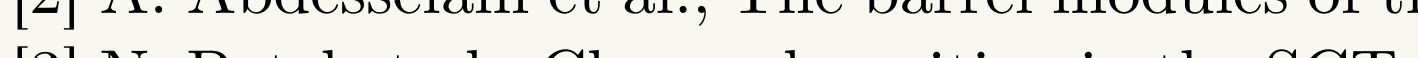
(3) N. Patel et al., Charge deposition in the SCT due to beamloss, ATLAS Note ATL-INDET-PUB-2013-002-
[4 F. C.

semiconductor tracker, Nucl. Instrum. Methods A552 292 (2005).
[5] A. Kuhl et al., ATLAS ABCD hybrid fatal charge dosage test, 2011 JINST 6 C12021.

[5] A. Kuhl et al,, ATLAS
[6] K. Hara et al., Beam spp
Methods A541 15 (2005).

[7] H.F.-W. Sadrozinski et al., P

$\rightarrow>$ Special thanks to J. Kaplon from the CERN microelectronics group for helpul consultations, C. Haber from LBL for providing the $\rightarrow$ Work supported by the U.S. Dept. of Energy under grant DE-FG02-13ER 41983 